\title{
Poisson statistics in the high temperature QCD Dirac spectrum
}

\author{
Tamás G. Kovács ${ }^{* \dagger}$ \\ Department of Physics, University of Pécs \\ H-7624 Pécs, Ifjúság útja 6, Hungary \\ E-mail: kgt@fizika.ttk.pte.hu

\section{Ferenc Pittler} \\ Department of Physics, University of Pécs \\ H-7624 Pécs, Ifjúság u. 6, Hungary \\ E-mail: pittlerferencegmail.com
}

At low temperature in the epsilon regime of QCD the low-end of the Dirac spectrum is described by random matrix theory. In contrast, there has been no similarly well established staistical description in the high temperature, chirally symmetric phase. Using lattice simulations we show that at high temperature a band of extremely localized eigenmodes appear at the low-end of the Dirac spectrum. The corresponding eigenvalues are statistically independent and obey a generalized Poisson distribution. Higher up in the spectrum the Poisson distribution rapidly crosses over into the bulk distribution predicted by the random matrix ensemble with the corresponding symmetry. My results are based on quenched lattice simulations with the overlap and the staggered Dirac operator done well above the critical temperature at several volumes and values of $N_{t}$. We also discuss the crucial role played by the fermionic boundary condition and the Polyakov-loop in this phenomenon.

The XXVIII International Symposium on Lattice Field Theory, Lattice2010

June 14-19, 2010

Villasimius, Italy

\footnotetext{
${ }^{*}$ Speaker.

${ }^{\dagger}$ Supported by OTKA Hungarian Science Fund grant 49652 and EU Grant (FP7/2007-2013)/ERC N 208740.
} 


\section{Introduction}

The lowest part of the spectrum of the QCD Dirac operator encodes important physical information concerning the low energy behavior of strongly interacting systems. In the so called epsilon regime of the low temperature phase the statistics of the lowest Dirac operator eigenvalues is described by chiral random matrix theory (RMT). This is a well established fact supported by analytic calculations in the corresponding low energy sigma model as well as extensive numerical lattice studies (see e.g. [1] for a summary of results and a list of original references). In contrast, above the finite temperature transition $\left(T_{c}\right)$ there is no generally accepted statistical description of the low end of the Dirac spectrum. Since in this regime there is no first principles analytic information available, to a first approximation the Dirac operator here can be regarded as a large fluctuating random matrix, with its size going to infinity in the thermodynamic limit.

From this perspective there are two possible types of fundamentally different extreme behavior the statistics of the lowest part of the spectrum can follow. If typical fluctuations cannot mix eigenmodes nearby in the spectrum, eigenmodes have to be localized, the corresponding eigenvalues are expected to be statistically independent and follow essentially Poisson statistics. If on the other hand, nearby eigenmodes in the spectrum can easily mix, they necessarily become delocalized and the spectrum is expected to obey random matrix statistics. Lattice simulations can decide which scenario is realized in nature.

Above $T_{c}$ chiral symmetry is restored and the density of eigenvalues of $D$, the order parameter of chiral symmetry breaking, vanishes. Random matrix theory (RMT) has specific predictions for the eigenvalue statistics around such a "soft edge" [2]. Lattice simulations, however, failed to reproduce the RMT predictions for the spectral statistics at the spectrum edge [3,4]. On the other hand, bulk random matrix statistics for full Dirac spectra above $T_{c}$ were verified previously [5]. Based on lattice simulations around the critical temperature, Ref. [6] suggested that the around the chiral transition at $T_{c}$ a gradual change of eigenvalue statistics at the edge occurs from RMT towards Poisson. Very recently, however, Ref. [7] argued that although low Dirac eigenmodes are localized, localization appears to be a finite volume artifact disappearing in the thermodynamic limit. If true, this would suggest RMT statistics for eigenvalues at the spectrum edge. Lattice results obtained so far are thus rather controversial.

In the present paper we provide some explanations for these apparent controversies and draw a clear picture of the eigenvalue statistics above $T_{c}$. The new ingredient in our analysis is that we study the eigenvalue statistics separately in different regions of the spectrum starting with the lowest eigenvalues and going upwards. Our main result is that the lowest part of the spectrum consists of localized, independent eigenmodes obeying Poisson statistics. Eigenmodes higher up in the spectrum gradually become more delocalized and at the same time the eigenvalue statistics crosses over to bulk random matrix statistics that was previously seen in lattice simulations. The phenomenon we report here is analogous to Anderson localization in conducting crystalline solids with disorder. In that case disorder can render electronic states at the band edge localized and non-conducting while states deep in the band can still remain conducting and delocalized. 


\section{Simulation details}

We performed simulations of the quenched $S U(2)$ gauge theory at a temperature well above the critical temperature. Here the Polyakov loop $Z(2)$ symmetry is spontaneously broken and for the quenched theory the two $Z(2)$ sectors are equivalent. In contrast, the Dirac spectrum is known to depend strongly on the Polyakov loop sector through the lowest Matsubara mode [8]. It is in fact the combination of the Polyakov sector and the antiperiodic quark boundary condition (b.c.) in the time direction that determines the lowest part of the Dirac spectrum. If this combination corresponds effectively to periodic b.c. (no twist), the spectral density does not vanish at zero. If on the other hand, the combined effective b.c. is antiperiodic (a twist of -1 ), the spectral density vanishes at zero and there might even be a gap in the spectrum there.

In the presence of light dynamical quarks with antiperiodic b.c. the small modes in the untwisted sector would suppress the determinant and only the positive Polyakov loop sector survives for large volumes. This is how the fermion action breaks the Polyakov loop $Z(2)$ symmetry above $T_{c}$ [9]. For this reason we used for our study only configurations in the positive Polyakov loop sector to mimic the most important effect of dynamical quarks on the Dirac spectrum.

We analyzed spectra of both the staggered and the overlap Dirac operator on $N_{t}=4$ and $N_{t}=6$ configurations with spatial volumes between $12^{3}-48^{3}$, all at the same physical temperature $T=$ $2.6 T_{c}$. The configurations were generated with the Wilson gauge action at $\beta=2.60$ and 2.725. The staggered and the overlap Dirac operator gave qualitatively similar results and both data sets support our main findings. More details of this study can be found in [10] for the overlap and in [11] for the staggered Dirac operator. In the following we shall present some results with both types of lattice Dirac operator.

\section{Localization of small modes}

At first we directly measured the localization of the eigenmodes in different regions of the low end of the spectrum. Instead of the most commonly used quantity, the inverse participation ratio (IPR), we used the quantity

$$
\mathscr{V}_{\psi}=\left[\sum_{x}\left(\psi^{\dagger} \psi(x)\right)^{2}\right]^{-1}
$$

for characterizing the localization of the normalized eigenmode $\psi . \mathscr{V}_{\psi}$ can be thought of as an approximate measure of the four-volume occupied by the eigenmode. This can be seen by considering an idealized eigenmode that is constant in a given subvolume $v$ of the total volume $V$ and zero elsewhere. We assume that at very high temperatures the eigenmodes are maximally spread in the (short) time direction and define a length scale

$$
d_{\psi}=\left[\frac{\mathscr{V}_{\psi}}{N_{t}}\right]^{1 / 3}
$$

measuring the linear spatial extension of the eigenmode $\psi$.

In Fig. 1 we plot how the average linear size of eigenmodes change as we go upwards in the spectrum starting from the lowest modes. It is apparent that in the lowest part of the spectrum the eigenmodes are very localized and their size is independent of the box size. Higher up in the 


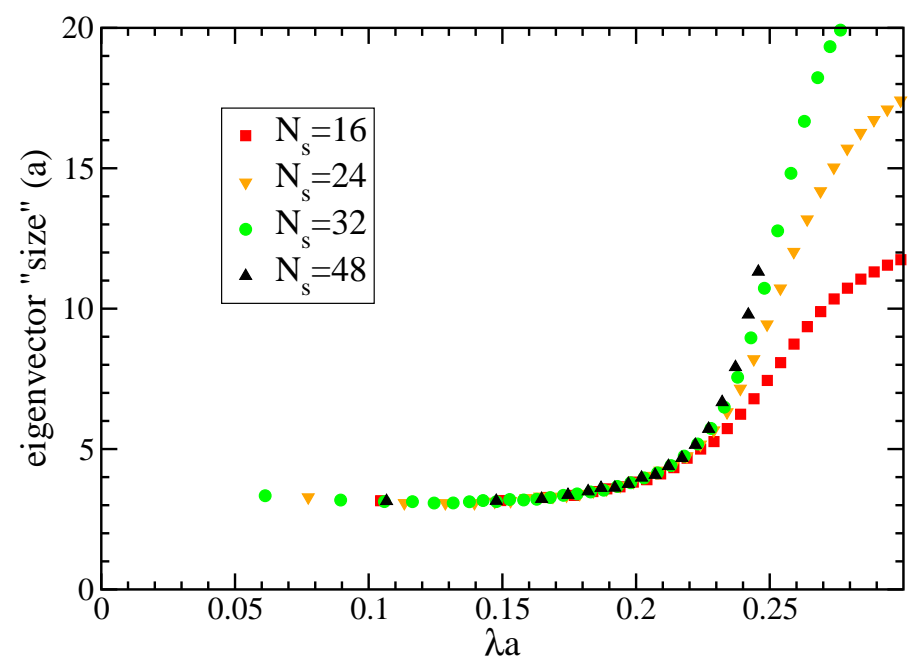

Figure 1: The average linear extension (in lattice units) of staggered eigenvectors, $d_{\psi}$, as a function of the corresponding eigenvalues. The different symbols correspond to spatial box sizes $N_{s}=16,24,32,48$. All the ensembles have $N_{t}=4$.

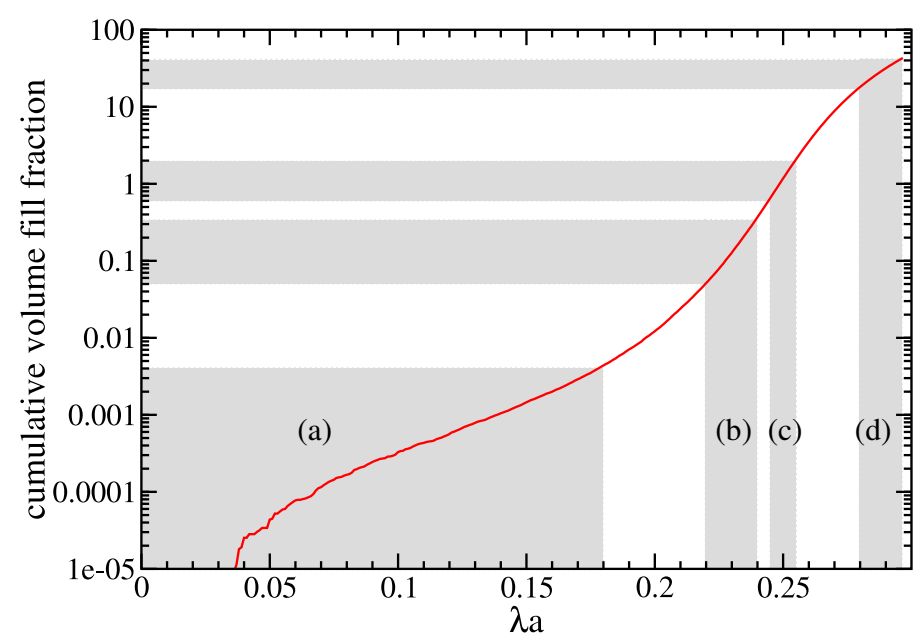

Figure 2: The cumulative volume fill fraction for the $24^{3} \times 4$ ensemble. The unfolded level spacing distribution will be computed separately in the four shaded spectral regions marked by (a)-(d) (see Fig. 4).

spectrum the eigenmodes spread out and they start to be constrained by the finite box. There is a rather sharp transition point between the two types of behavior, analogous to the mobility edge separating conducting and non-conducting electron states in disordered conductors.

Besides spatial localization, the other quantity affecting the mixing of nearby modes in the spectrum, is their density. These two effects together determine how much spatial overlap nearby modes in the spectrum typically have. A useful quantity reflecting this can be defined by summing the participation ratios of all modes up to a given point $\lambda$ in the spectrum. We call this the volume fill fraction (VFF). It is essentially the fraction of the total four-volume occupied by modes up to a given eigenvalue $\lambda$. In Fig. 2 we show this as a function of the eigenvalue $\lambda$. In the lowest part of the spectrum where the spectral density is small and modes are localized, the VFF is much less 


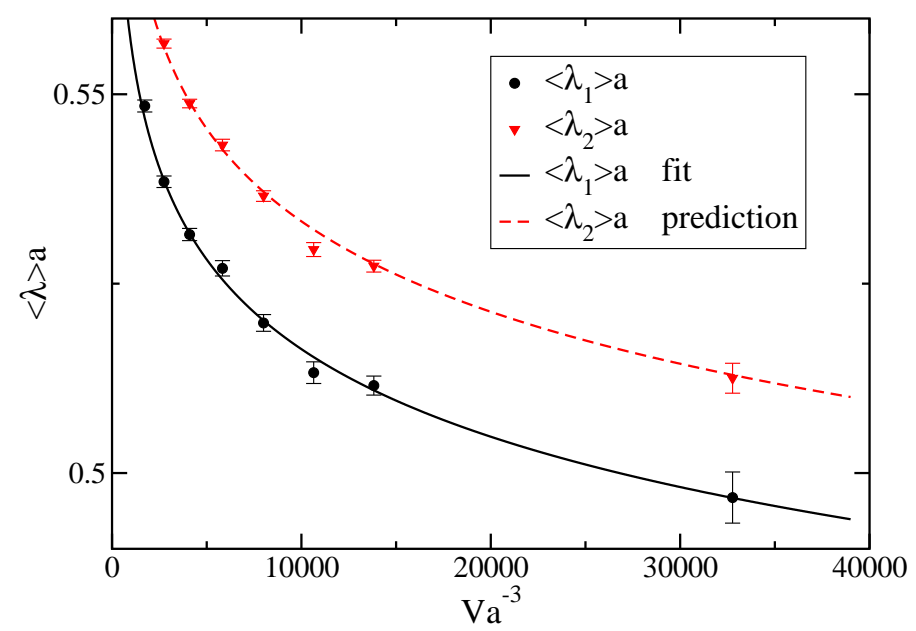

Figure 3: The volume dependence of the smallest two eigenvalues of the overlap Dirac operator for $N_{t}=4$ and Wilson $\beta=2.6$. The solid line is a two parameter fit $(\alpha, C)$ to the analytic prediction based on Poisson statistics and power law spectral density. The dashed curve is not a fit, it is the prediction for the second smallest eigenvalue based on the already fitted values of $\alpha$ and $C$.

than unity allowing for eigenmodes to occur without substantial spatial overlap. If the eigenmodes are really produced in independent subvolumes the level statistics is expected to be Poissonian. In contrast, higher up in the spectrum the VFF becomes much bigger than unity and eigenmodes there must overlap. Here the eigenvalue statistics is expected to be described by random matrix theory.

There are several ways of explicitly checking these expectations on lattice Dirac spectra. The Poisson and random matrix statistics pertain to two different universal ways the eigenvalue fluctuations can be correlated locally in the spectrum. An additional, non-universal feature of the spectrum is the spectral density, $\rho(\lambda)$. In the case of the overlap operator the spectral density of the low modes is numerically found to be well described by a simple power law $\rho(\lambda)=C \lambda^{\alpha}$. Using this and assuming statistically independent spectral fluctuations for the lowest eigenvalues (Poisson statistics) we can derive detailed predictions for the distribution of the lowest first, second, etc. eigenvalues and their dependence on the spatial volume of the lattice. As an illustration, in Fig. 3 we compare the spatial volume dependence of the lowest two eigenvalues with the analytic predictions based on Poisson statistics and the power law spectral density. The solid line is a two-parameter fit of this analytic form to the average lowest eigenvalue. The dashed line is a parameter-free prediction for the average of the second smallest using the already fitted parameters. Both the fit and the parameter-free prediction describe the data correctly, suggesting that the smallest two eigenvalues indeed obey Poisson statistics.

For the staggered Dirac operator we did not find a simple analytic description of the spectral density, but we had much more eigenvalues per configuration. This allowed us to compute the so called unfolded level spacing distribution. Unfolding is a standard way in random matrix theory to locally rescale the spectrum in order to get rid of the dependence on the non-universal spectral density. If the eigenvalues are statistically independent, after unfolding the levels are described by a simple Poisson distribution with exponentially distributed level spacings. If on the other hand, the original spectrum follows random matrix statistics, the distribution of the unfolded level spacings 
(a)

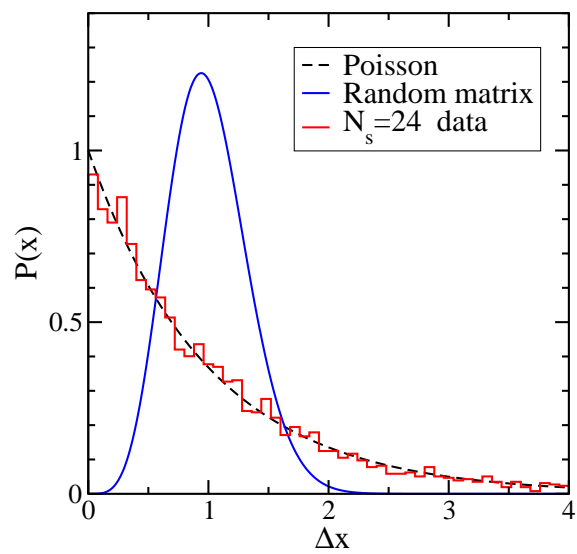

(c)

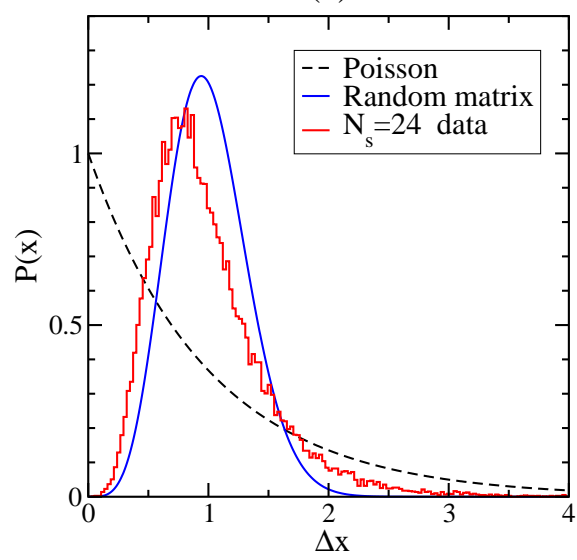

(b)

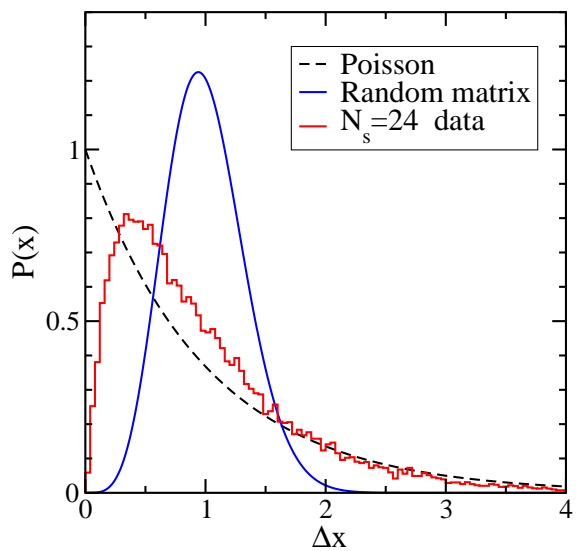

(d)

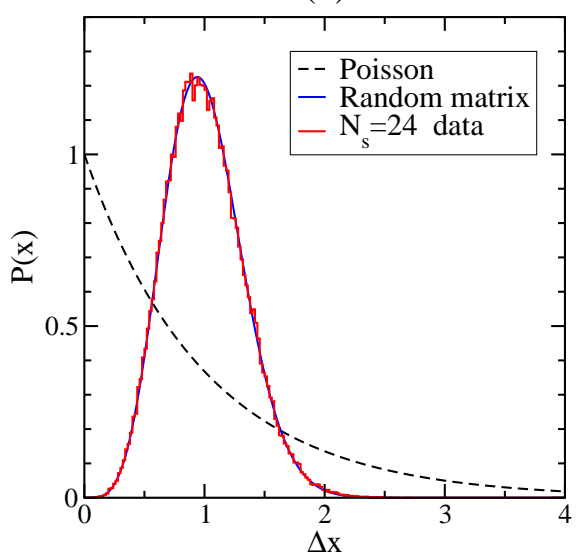

Figure 4: The panels show the unfolded level spacing distribution in different regions of the spectrum. The labeling (a)-(d) corresponds to the regions indicated in Fig. 2 with the shaded areas. The curved lines are the exponential distribution and the Wigner surmise.

is described by the so called Wigner surmise of the given random matrix ensemble [1]. In the case of the staggered Dirac operator the corresponding ensemble is the chiral symplectic ensemble.

In Fig. 4 we show the unfolded level spacing distribution of the eigenvalues in four different regimes of the spectrum. Also shown in each figure is the universal (parameter-free) prediction based on Poisson and random matrix statistics respectively. It is apparent that the lowest part of the spectrum obeys Poisson statistics and that going up in the spectrum this gradually crosses over to random matrix statistics.

An important question is whether this phenomenon survives the thermodynamic and the continuum limit. When scaling up the spatial volume one expects that the number of "modes of a given statistics" scales proportionally to the volume. This can be most simply checked for the eigenvalues of intermediate statistics. In Fig. 5(a) we plotted the level spacing distribution of eigenvalues 10-20 in a spatial volume of $24^{3}$ and that of the scaled up percentage of eigenvalues $24-47$ in a volume of $32^{3}$. The distributions on the two volumes appear to be the same intermediate distribution demonstrating that the number of eigenvalues of the same statistics scales with the volume. 
(a)

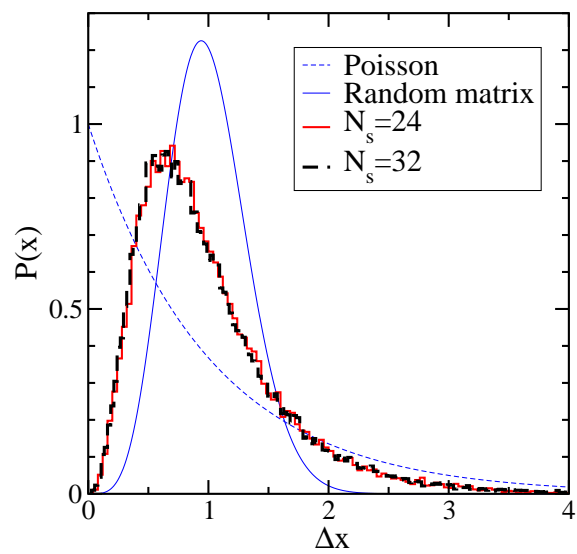

(b)

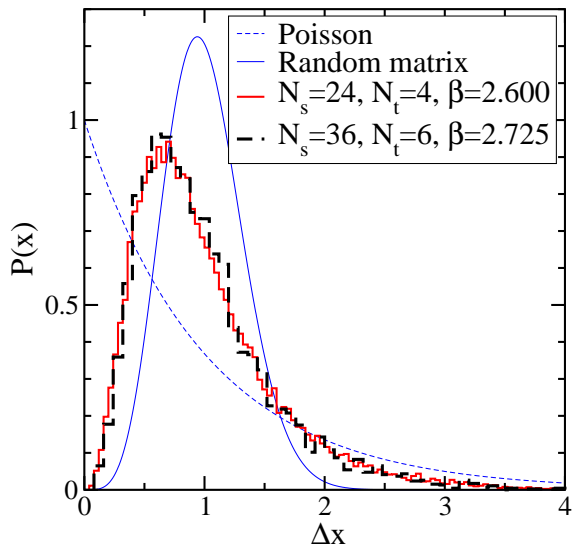

Figure 5: The unfolded level spacing distribution for two different spatial volumes at fixed $N_{t}$ (a) and for two different $N_{t}$ 's at fixed physical temperature and physical volumes (b).

To assess what happens in the continuum limit, in Fig. 5(b) we show level spacing statistics for the same physical temperature and three-volume with two different lattice spacings (corresponding to $N_{t}=4$ and $N_{t}=6$. For both cases we plotted the statistics based on eigenvalues 10-20. Although the difference in lattice volumes in lattice units is more than a factor of 3, the two distributions seem to be identical. This shows that the number of eigenvalues with a given statistics depends on the physical volume, not on the volume in lattice units. The lowest, Poisson distributed modes might thus be associated with some physical objects in the gauge field background, the physical density of wich is constant in the continuum limit.

\section{References}

[1] J. J. M. Verbaarschot and T. Wettig, Ann. Rev. Nucl. Part. Sci. 50, 343 (2000) [arXiv:hep-ph/0003017].

[2] P. J. Forrester, Nucl. Phys. B402[FS], 709 (1993)

[3] F. Farchioni, P. de Forcrand, I. Hip, C. B. Lang and K. Splittorff, Phys. Rev. D 62, 014503 (2000) [arXiv:hep-lat/9912004];

[4] P. H. Damgaard, U. M. Heller, R. Niclasen and K. Rummukainen, Nucl. Phys. B 583, 347 (2000) [arXiv:hep-lat/0003021].

[5] R. Pullirsch, K. Rabitsch, T. Wettig and H. Markum, Phys. Lett. B 427, 119 (1998) [arXiv:hep-ph/9803285].

[6] A. M. Garcia-Garcia and J. C. Osborn, Phys. Rev. D 75, 034503 (2007) [arXiv:hep-lat/0611019].

[7] R. V. Gavai, S. Gupta and R. Lacaze, Phys. Rev. D 77, 114506 (2008) [arXiv:0803.0182 [hep-lat]].

[8] E. Bilgici et al. arXiv:0906.3957 [hep-lat].

[9] T. G. Kovacs, PoS LATTICE2008, 198 (2008) [arXiv:0810.4763 [hep-lat]].

[10] T. G. Kovacs, Phys. Rev. Lett. 104, 031601 (2010) [arXiv:0906.5373 [hep-lat]].

[11] T. G. Kovacs and F. Pittler, arXiv:1006.1205 [hep-lat]. 